\title{
Towards eradication: adopting a community- directed intervention strategy in the prevention and management of malaria in Zimbabwe
}

\begin{abstract}
Zimbabwe continues to struggle to eliminate malaria despite the availability of several global health1 initiatives to manage the disease. There is little empirical evidence on how to achieve the integration of community-level interventions in the context of malaria prevention and management, and public health in general. This monograph traverses the dangers posed by malaria as a disease within the Zimbabwean context. It proposes and encourages the conducting of theory-based research to generate appropriate data that can be used in the implementation of strategies aimed at eventual eradication of malaria and management and control of other diseases.
\end{abstract}

Volume 2 Issue 3 - 2018

\author{
Muchanyerei Babbot \\ Honours in Social Work, University of Zimbabwe, Zimbabwe
}

Correspondence: Honours in Social Work, University of Zimbabwe, MSW, University of Pretoria, Johannesburg, South Africa, Tel 0027630320089, Email babbotm@webmail.co.za

Received: February 27, 2018 | Published: May 15, 2018

Keywords: malaria, prevention and management, community-directed intervention, Zimbabwe

\section{Introduction}

Zimbabwe continues to agonise over malaria with the disease wreaking havoc in the Southern African country. For instance, in the first nine weeks of 2017 the country recorded over 89000 infections and about 151 deaths. ${ }^{2}$ Malaria is both a health and socio-economic issue $^{3}$ as it does not only cause illness and premature death, but also impact negatively on social development as well as productivity (Ministry of Health and Child Care, ${ }^{4}$ Fortunately, the disease is preventable. This article explores malaria as an epidemic in Zimbabwe and proposes the adoption of community-directed interventions as a measure to prevent and curb the impact of the disease. The argument is that the empowerment and inclusion of communities will go a long way in addressing the epidemic.

\section{Background}

Malaria remains a grave global and public health burden causing high morbidity and mortality. It affects more than 100 countries, with an annual incidence of more than 200 million cases and nearly one million deaths. ${ }^{5,6}$ The parasitic disease continues to be a veritable concern in the developing world, particularly in Africa. ${ }^{7}$ Despite a significant global decline in both incidence and deaths due to the availability of some of the best interventions, malaria is still a ubiquitous killer especially among children and pregnant women who account for more than eighty percent in the number of deaths. ${ }^{6-8}$

\section{The Zimbabwean tale}

In Zimbabwe, malaria is the third leading cause of illness and mortality, after HIV/AIDS and tuberculosis. ${ }^{9,10}$ Reports also indicate that after HIV and AIDS, malaria is the biggest killer of children under five, pregnant women and new-borns in the country. ${ }^{11}$ According to David Parirenyatwa, the Zimbabwean minister of Health and Child Care, over half of the population is at risk of contracting malaria with more than 400,000 malaria cases among all age groups each year, reported in the country. $\mathrm{WHO}^{6}$ puts the reported annual malaria episodes at close to 1,5 million with average of 1000 deaths.
Zimbabwe has a long history of malaria epidemics. The primary malaria endemic zones are in the northern and eastern regions bordering Mozambique and Zambia. There are several districts that are malarial with some of them categorised as high-burden malaria areas. The worst affected districts are in the provinces of Manicaland, Mashonaland East and Mashonaland Central. For instance, approximately eighty-three percent of all malaria cases and fifty percent of all malaria-related deaths in 2014 originated from these three provinces, with forty-two percent of all cases and twenty-six percent of all deaths recorded in Manicaland province. ${ }^{4}$

Although statistics continuously show a decline in malaria incidences in Zimbabwe, the disease remains a major challenge in certain districts particularly in seven provinces of the country's ten provinces. With a majority of the population surviving on less than a dollar per day especially in the rural areas, poverty has resulted in the perpetuation of the disease. This is mainly so because malaria, as a disease and public health challenge, reflects an extremely complex set of interactions between the parasite, the human host, and the vectors responsible for transmission. ${ }^{4}$ These interactions are fostered by the environmental, socio-economic and behavioural circumstances in which most people find themselves.

\section{Why community-directed interventions?}

Despite the proliferation of several global health initiatives and the subsequent improvement in the delivery of selected health interventions, many priority interventions including those directed against malaria still have unacceptably low coverage, especially in Africa. ${ }^{3}$ The $\mathrm{WHO}^{3}$ bemoans the fragmentation of several health initiatives, and calls for integration of particularly those strategies and interventions that improve access and encourage community participation. Madon et al..$^{12}$ argue that many disease control measures that are simple, affordable and effective, have had only limited impact on the burden of disease due to their inadequate distribution in poor and remote communities. 
The community-directed intervention (CDI) is such one strategy, which if appropriately implemented, is both effective and efficient for integrated delivery of appropriate health interventions. According to $\mathrm{WHO}^{3} \mathrm{CDI}$ is a health intervention that is undertaken at the community level under the direction of the community itself. A range of possible interventions are introduced in a participatory manner thereby ensuring community ownership from the onset.

Over half of the Zimbabwean population of about 13 million people is at risk of contracting malaria. This is happening at a time the anopheles mosquito, the vector that transmits the disease, is increasingly resisting the commonly used residual spray. Zimbabwe cannot, therefore, make the list of African countries expected to reach the year 2020 target of being malaria-free. It is believed that only six African countries namely, Algeria, Botswana, Cape Verde Islands, Comoros, South Africa and Swaziland could be malaria-free by Malaria Day 2020.

Following the findings of its 2005-2007 study in selected African countries, $\mathrm{WHO}^{3}$ recommended that $\mathrm{CDI}$ approaches be adopted for integrated, community-level delivery of appropriate health interventions especially for malaria. Consequently, there is need to fulfil this recommendation through the gathering of empirical data on the feasibility and effectiveness of such interventions in the country.

\section{A theory-based approach}

Tung et al. ${ }^{13}$ observe that a theory-based assessment that would allow healthcare professionals to develop more effective and costeffective programmes is immediately needed. It suffices to say, nonetheless, that the selection of a theory or theories over another remains a choice of the researcher although one theory or theories may be more relevant than the other in the exploration and explication of a given phenomenon.

Various studies have established that there are challenges to working with patients to change their behaviours to reduce the likelihood of contracting a disease such as malaria, and that some of the challenges are highlighted in the dimension of the Health Belief Model (HBM). The HBM is "by far the most commonly used theory in health education and health promotion". ${ }^{14}$ The original HBM posits that health behaviour is determined by personal beliefs or perceptions about a disease and the strategies available to curtail its manifestation. It further postulates that people will take action to prevent illness if they regard themselves to be susceptible to a condition (perceived susceptibility); if they believe it would have potentially serious consequences (perceived severity); if they believe that a particular course of action available to them would reduce the susceptibility or severity or lead to other benefits (perceived benefits); if they perceive few negative elements related to the health action (perceived barriers); and if they believe that one can successfully complete the behaviour of interest despite considered barriers (perceived self-efficacy). The HBM therefore advances that health messages will achieve optimum change if they successfully target these constructs, namely perceived threat, barriers, benefits and self-efficacy. ${ }^{15}$

Another approach, the Empowerment Theory is one of those social theories that underscore participation and empowerment as well as connecting the individual and collective at micro and macro levels. ${ }^{16}$ It borrows and encompasses certain philosophies from related social theories such as Radical and Democratic theories, Community Development, Feminist and Anti-Discriminatory theories, Strength-
Based theories, and the Psychodynamic theory, which in turn makes it a very strong approach to social change. ${ }^{17}$ This theory involves processes and outcomes. ${ }^{18}$ The processes include participation in community organisations, collective decision-making and action, and shared leadership. Outcomes include resource mobilisation skills, development of organisational networks, evidence of pluralism, and accessible community resources. Social inclusion and participation, which are tenets of empowerment, enable people and communities to fully participate in society and empower poor and marginalised people to have a voice in decisions that affect their lives as well as ensuring that they enjoy equal access to markets, services, and political, social and physical spaces. ${ }^{19}$

It has been observed that effective behaviour change is difficult to initiate and sustain even when communities are informed well about simple means of health promotion or harm reduction.$^{20}$ Knowledge about the threat and potential severity of malaria and ways to prevent and manage the disease is important but inadequate on its own. A combination of this knowledge and the wherewithal to address the endemic is needed. The application and amalgamation of the HBM and Empowerment Theory is therefore believed to bring an important dimension and insight on how community-directed interventions may be effective in the prevention and management of disease in general and malaria in particular.

\section{Conclusion and implications for social workers}

This article has discussed the prevalence and impact of malaria in Zimbabwe. It has recommended the adoption of community-directed interventions as a prevention and management strategy. It was argued that the adoption of such a strategy should be done within theorybased research. As a social worker also, the author calls upon social work practitioners to be actively involved in public health issues in general and in interventions aimed at preventing malaria in particular. Social workers play a critical role in maintaining a nation's social fabric and protecting vulnerable members of society. ${ }^{21}$ As such, their involvement in public health issues such as disease prevention and management, community conscientization, and research is of paramount importance.

\section{Acknowledgements}

None.

\section{Conflict of interest}

The author declares that there is no conflict of interest.

\section{References}

1. Global health. USA: USAID; 2017. $220 \mathrm{p}$

2. Bridge Mananavire. Malaria continues to wreak havoc. USA: Daily News; 2017.

3. World Health Organisation. Maximising positive synergies collaborative group: An assessment of interactions between global health initiatives and country health systems. Lancet. 2009;373(1):2137-2169.

4. National Helath Profile. Zimbabwe: Zimstat; 2014. 198 p.

5. Hall BF, Fauci AS. Malaria control, elimination, and eradication: The role of the evolving biomedical research agenda. The Journal of Infectious Diseases. 2009;200(11):1639-1643. 
6. World Health Organisation. World Malaria Report. Geneva: WHO; 2011 $186 \mathrm{p}$.

7. Tobgay T, Deki Pem, Ugyen Dophu, et al. Community-directed educational intervention for malaria elimination in Bhutan: Quasi experimental study in malaria endemic areas of Sarpang district. Malaria Journal. 2013;12:132.

8. Laishram DD, Patrick L Sutton, Nutan Nanda, et al. The complexities of malaria disease manifestations with a focus on asymptomatic malaria. Malaria Journal. 2012;11(29):1-15.

9. Mutenga T. Zimbabwe agonises over malaria. Zimbabwe: The Financial Gazette; 2016.

10. Global health. USA: USAID; 2017.220 p

11. Chipunza P. Malaria cases on the rise. Scotland: The Herald; 2017.

12. Madon T, Hofman KJ, Kupfer L, et al. Public health: Implementation Science. Science. 2007;318(5857):1728-1729.

13. Tung WC, Nguyen DH, Tran DN. Applying the trans-theoretical model to cervical cancer screening in Vietnamese-American women. Journal of International Nursing. 2008;55(1):73-80.

14. Hayden J. Introduction to health behaviour theory. $2^{\text {nd }}$ ed. Burlington: Jones \& Bartlett Learning; 2014. 1-8 p.
15. Jones CL, Jensen JD, Scherr CL, et al. The Health Belief Model as an explanatory framework in communication research: Exploring parallel, serial and moderated mediation. Health Communication. 2015;30(6):566576.

16. Sadan E. Empowerment and community planning: Theory and practice of people-focused social solutions. Tel Aviv: Hakibbutz Hameuchad Publishers; 1997. $350 \mathrm{p}$.

17. Payne M. Modern Social Work Theory. $3^{\text {rd }}$ ed. Illinois: Lyceum Book Inc; 2005. 366 p.

18. Van Dyk A. HIV and AIDS education, care and counselling. $4^{\text {th }}$ ed. Cape Town: Pearson Education South Africa Pty Ltd; 2012. 512 p.

19. Social inclusion. USA: World Bank; 2013.

20. Panter Brick C, Clarke SE, Lomas H, et al. Culturally compelling strategies for behaviour change: A social ecology model and case study in malaria prevention. Social Science Medicine. 2006;62(11):2810-2825.

21. Muchanyerei B. Apathy of fear? The role of social workers in Zimbabwe's political discourse. African Journal of Social Work. 2017;7(2):60-64. 